\title{
Interactive Axial-Moment Dynamic Buckling of Beams by Spline Finite Element
}

\author{
Hao Yang, Sai-Huen Lo, and Yee Tak Leung
}

\begin{abstract}
This paper aims to investigate the dynamic buckling problems of linear beam system with initial axial force and uniform moment by the developed spline finite element method, which is employed to obtain the dynamic stiffness matrix. Second order effects of the axial force and moment are considered. The moment-frequency interaction diagrams in uniform axial force and moment are plotted. The effects of warping rigidity, torsional rigidity, axial tension and compression on moment buckling are investigated in detail. The spline finite element method is proved to be very efficient for the present problems and many interaction diagrams can be plotted easily.
\end{abstract}

Index Terms-Beam, dynamic buckling, spline finite element.

\section{INTRODUCTION}

B-splines were first introduced by Schoenberg [1], [2] in 1946 for solving certain data-fitting problems, especially in the case of uniform knots. Spline functions are convenient approximation and interpolation tools due to their continuity, capacity to handle local phenomena, good accuracy and convergence characteristics. Second-order linear buckling formulation is an important part in getting the symmetric tangential stiffness matrix due to initial stress in a nonlinear analysis [3]. Kim et al. [4] formed the static and dynamic stiffness matrices for the flexural-torsional dynamic buckling analysis of nonsymmetric cross-sectional thin-walled beams using power series method. Leung applied power series method to investigate the dynamic axial-moment buckling [5] and axial-torsional buckling [6] of linear framed structures. Yang and Leung [7] developed the spline finite element method to investigate the dynamic axial-torsional buckling of beams. The present paper investigates the interactive axial-moment dynamic buckling of beams of double symmetric cross-sections by spline finite element method. Second order effects of the axial force and moment are considered. The effects of shear lag, local buckling, and distortional buckling are not included. The spline finite element method is employed to obtain the dynamic stiffness matrix. The moment-frequency interaction diagrams in uniform axial force and moment are plotted. The effects of

Manuscript received March 4, 2013; revised May 21, 2013. The financial support from the HKSAR GRF Grant to the research project HKU715110E on "Drifted based seismic fragility analysis of high-rise RC buildings with transfer structures" is greatly acknowledged.

Hao Yang and Sai-Huen Lo are with the Department of Civil Engineering, The University of Hong Kong, Pokfulam, Hong Kong (e-mail: hollis.yang.hao@gmail.com, hreclsh@hku.hk).

Tee Tak Leung is with the Department of Civil and Architectural Engineering, College of Science and Engineering, City University of Hong Kong (e-mail: andrew.leung@cityu.edu.hk). warping rigidity, torsional rigidity, axial tension and compression are investigated. The method is proved to be very efficient for the present problems.

\section{ENERGY EQUATIONS}

Classical assumptions are applied to a doubly symmetric beam of cross-sectional area $A$, as shown in Fig. 1, with initial stresses due to bending moment $M$ along x axis and axial force $P$ along the centroid $z$ axis. The density of material is indicated by $\rho$. The displacements, $u_{x}, u_{y}$ and $u_{z}$, of a generic point at cross-section $z$ can be defined by the twist angle $\theta$ and the displacement $u$ along $x$ axis of the centroid of cross-section $z$ is as

$$
\mathbf{u}(x, y, z, t)=\left\{\begin{array}{l}
u_{x} \\
u_{y} \\
u_{z}
\end{array}\right\}=\left\{\begin{array}{c}
u-y \theta \\
v+x \theta \\
-x u^{\prime}-y v^{\prime}
\end{array}\right\}
$$

where, a prime denotes differentiation with respect to $z$. The axial deformation will not be considered. The initial normal stress due to the axial force $P$, bending moments $M$ is [8] $\tau_{z z}^{0}=$ $P / A-M y / I_{x}+N x / I_{y}$, in which $I_{x}$ and $I_{y}$ are the principal moments. This paper aims to develop the spline element method for interactive buckling of beams subject to axial load and moments, we shall only consider the case in $2 \mathrm{D}$ for simple presentation.

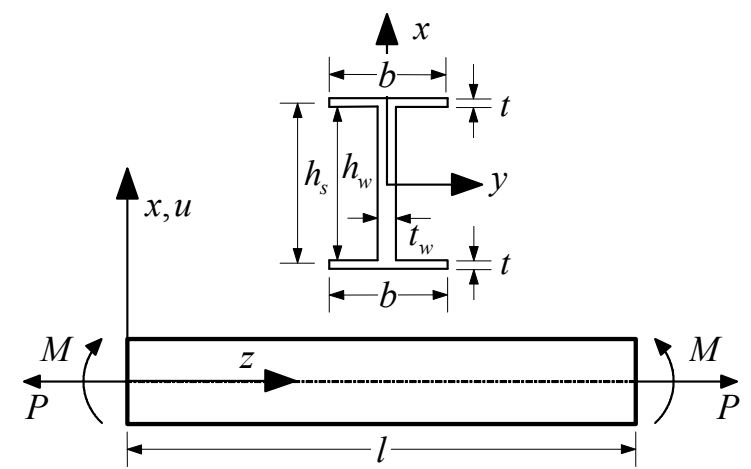

Fig. 1. A doubly symmetric thin beam of uniform cross-section

The strain energy due to initial axial stress $U_{\sigma}$, the linear strain energy $U_{e}$ and the kinetic energy $T$ are respectively given by

$$
\begin{gathered}
U_{\sigma}=\frac{1}{2} \int \tau_{z z}^{0} \mathbf{u}^{\prime T} \mathbf{u}^{\prime} d V=\frac{1}{2} \int_{0}^{l}\left(P u^{\prime 2}+2 M u^{\prime} \theta^{\prime}\right) d z \\
U_{e}=\frac{1}{2} \int_{0}^{l}\left(G I_{t} \theta^{\prime 2}+E I_{y} u^{\prime \prime 2}+E I_{w} \theta^{\prime \prime 2}\right) d z
\end{gathered}
$$




$$
T=\frac{1}{2} \int_{0}^{l}\left(\rho A \dot{u}^{2}+\rho I_{p} \dot{\theta}^{2}\right) d z .
$$

where, $I_{p}=I_{x}+I_{y}$ is the polar moment; $G I_{t}, E I_{y}$ and $E I_{w}$ are the tortional rigidity, the flexural rigidity and the warping rigidity, respectively.

\section{DisPlaCEMENT FIELD}

We denote the coordinates of the node points for splines on the interval $[0,1]$ by $z_{0}, z_{1}, \ldots, z_{n}$, which discretize the beam into $n$ equi-sized sections with uniform span $h$, where $h=z_{i+1}-z_{i}$ and $z_{i}=z_{0}+i h, i=0,1, \ldots, n-1$. Six auxiliary nodes, $z_{-3}<z \quad{ }_{-2}<z_{-1}$ and $z_{n+1}<z_{n+2}<z_{n+3}$, are necessary. The displacements $u(z, t)$ and $\theta(z, t)$ are assumed to be approximated by

$$
\begin{aligned}
\left\{\begin{array}{l}
u(z, t) \\
\theta(z, t)
\end{array}\right\} & =\left\{\begin{array}{l}
\sum_{i=-1}^{n+1} \varphi_{i}(z) \alpha_{i}(t) \\
\sum_{i=-1}^{n+1} \varphi_{i}(z) \beta_{i}(t)
\end{array}\right\}=\left\{\begin{array}{l}
\boldsymbol{\varphi}(z) \boldsymbol{\alpha}(t) \\
\boldsymbol{\varphi}(z) \boldsymbol{\beta}(t)
\end{array}\right\} \\
& =\left[\begin{array}{cc}
\boldsymbol{\varphi}(z) & \mathbf{0} \\
\mathbf{0} & \boldsymbol{\varphi}(z)
\end{array}\right]\left\{\begin{array}{l}
\boldsymbol{\alpha}(t) \\
\boldsymbol{\beta}(t)
\end{array}\right\}=\boldsymbol{\Phi}(z) \mathbf{q}(t)
\end{aligned}
$$

The cubic B-spline basis functions $\varphi_{i}(z), i=-1,0,1, \ldots, n-1$, $n, n+1$, are defined by

$$
\varphi_{i}(z)=\frac{1}{6 h^{3}} \begin{cases}0 & z<z_{i-2} \\ \left(z-z_{i-2}\right)^{3} & z_{i-2} \leq z \leq z_{i-1} \\ h^{3}+3 h^{2}\left(z-z_{i-1}\right) & z_{i-1} \leq z \leq z_{i} \\ +3 h\left(z-z_{i-1}\right)^{2}-3\left(z-z_{i-1}\right)^{3} & \\ h^{3}+3 h^{2}\left(z_{i+1}-z\right) & z_{i} \leq z \leq z_{i+1} \\ +3 h\left(z_{i+1}-z\right)^{2}-3\left(z_{i+1}-z\right)^{3} & z_{i+1} \leq z \leq z_{i+2} \\ \left(z_{i+2}-z\right)^{3} & z>z_{i+2} \\ 0 & \end{cases}
$$

\section{EQUATIONS OF MOtION}

To reduce the number of variables, it is advantageous to non-dimensionalize the variables in $T, U_{e}$ and $U_{\sigma}$, by $\bar{z}=z / l$, thus

$$
\begin{gathered}
U_{\sigma}=\frac{E I_{y}}{2 l^{3}} \int_{0}^{1}\left(\bar{P} u^{\prime 2}+2 \bar{M} u^{\prime} \bar{\theta}^{\prime}\right) d \bar{z} . \\
U_{e}=\frac{E I_{y}}{2 l^{3}} \int_{0}^{l}\left(g \bar{\theta}^{\prime 2}+u^{\prime \prime 2}+\kappa \bar{\theta}^{\prime \prime 2}\right) d \bar{z} . \\
T=\frac{E I_{y}}{2 l^{3}} \int_{0}^{1} \frac{\lambda}{\omega^{2}}\left(\dot{u}^{2}+\dot{\bar{\theta}}^{2}\right) d \bar{z} .
\end{gathered}
$$

where, $\bar{P}=P l^{2} / E I_{y}, \bar{M}=M l^{2} / r E I_{y}, \bar{\theta}=r \theta, \kappa=E I_{w} / r^{2} E I_{y}, g=$ $G I_{t} l^{2} / r^{2} E I_{y}, \lambda=\omega^{2} \rho A l^{4} / E I_{y}, r^{2}=I_{p} / A$. According to the Hamilton's principle [9], one has the equations of motion, as follows

$$
\begin{aligned}
\lambda \mathbf{M} \ddot{\mathbf{q}}(t) / \omega^{2}+\mathbf{K q}(t) & \\
& +\left[\mathbf{K}_{\sigma g}+\mathbf{K}_{\sigma P}(P)+\mathbf{K}_{\sigma M}(M)\right] \mathbf{q}(t)=0
\end{aligned}
$$

For free vibration problem, (10) can be expressed as

$$
\mathbf{K}_{D} \overline{\mathbf{q}}=\left[\mathbf{K}+\mathbf{K}_{\sigma g}+\mathbf{K}_{\sigma P}(P)+\mathbf{K}_{\sigma M}(M)-\lambda \mathbf{M}\right] \overline{\mathbf{q}}=\mathbf{0}
$$

where, $\mathbf{M}, \mathbf{K}$ and $\mathbf{K}_{\sigma g}+\mathbf{K}_{\sigma P}(P)+\mathbf{K}_{\sigma M}(M)$ are the mass matrix, stiffness matrix, and initial stress matrix, respectively. $\mathbf{K}_{D}$ is the dynamic stiffness matrix. For nontrivial solution, $\operatorname{det}\left(\mathbf{K}_{D}\right)$ $=0$, which presents the relation between $\lambda, P$, and $M$ when buckling occurs. In subsequent studies, $\lambda^{1 / 4}, P^{1 / 2}$, and $M^{1 / 2}$ will be used as the primary parameters so that the spread of the curves is more uniform.

\section{NuMERICAL EXAMPLES}

Consider an I-section cantilever beam as shown in Fig. 1. The flange width $b=4 \mathrm{~cm}$, the flange thickness $t=0.5 \mathrm{~cm}$, the web depth $h_{w}=10 \mathrm{~cm}$, the web thickness $t_{w}=0.7 \mathrm{~cm}$, the distance between the shear centers of the flanges $h_{s}=11 \mathrm{~cm}$. $l=100 \mathrm{~cm}, \quad A=11 \mathrm{~cm}^{2}, E=2.1 \times 10^{7} \mathrm{~N} / \mathrm{cm}^{2}, \quad G=8.4 \times 10^{6} \mathrm{~N} / \mathrm{cm}^{2}$, $I_{x}=5.61 \mathrm{~cm}^{4}, \quad I_{y}=168.66 \mathrm{~cm}^{4}, \quad I_{p}=174.27 \mathrm{~cm}^{4}, I_{t}=1.4767 \mathrm{~cm}^{4}$, $I_{w}=161.3333 \mathrm{~cm}^{6}, r^{2}=15.8427 \mathrm{~cm}^{2}, \kappa=0.06038, g=2.2106$.

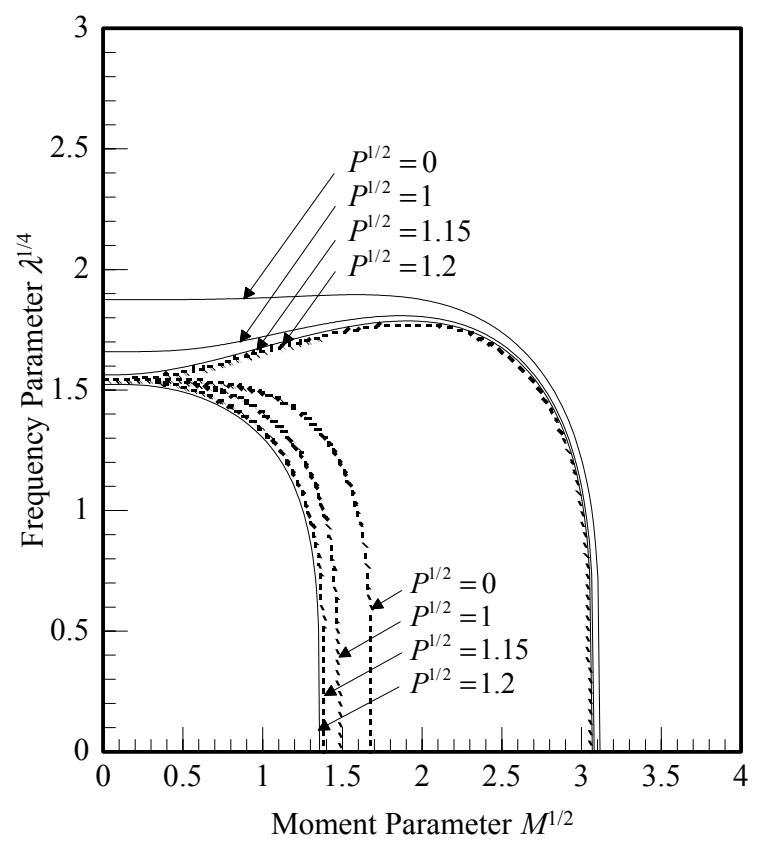

Fig. 2. Moment buckling under axial compression (...warping major branch, — bending major branch)

Fig. 2 presents that axial compression decreases the bending natural frequency while the warping natural frequency is not affected. On the other hand, it decreases the static bending buckling moment. When the axial compression parameter $\mathrm{P} 1 / 2$ is increased from 1.15 to 1.2 , the major bending branch crosses the major torsion-warping branch. The major bending branch and the major torsion-warping branch exchange so that bending buckling moment parameter M1/2 decreases suddenly from 3.0667 to 1.3569. Note that the slope of the interactive curves in both 
bending and torsion-warping are not monotonic.

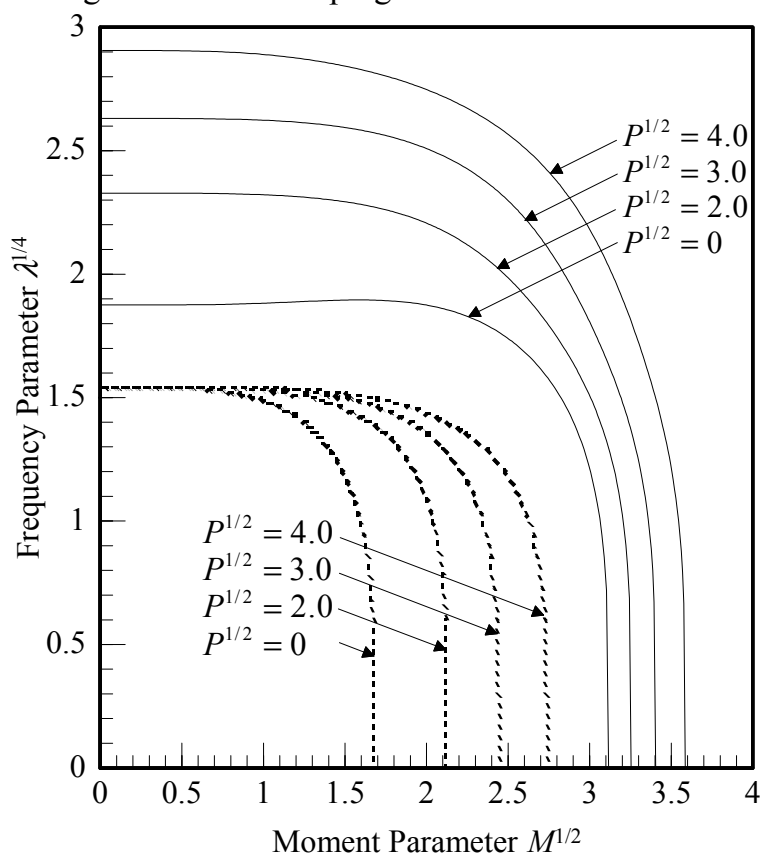

Fig. 3. Moment buckling under axial tension (...warping major branch, bending major branch)

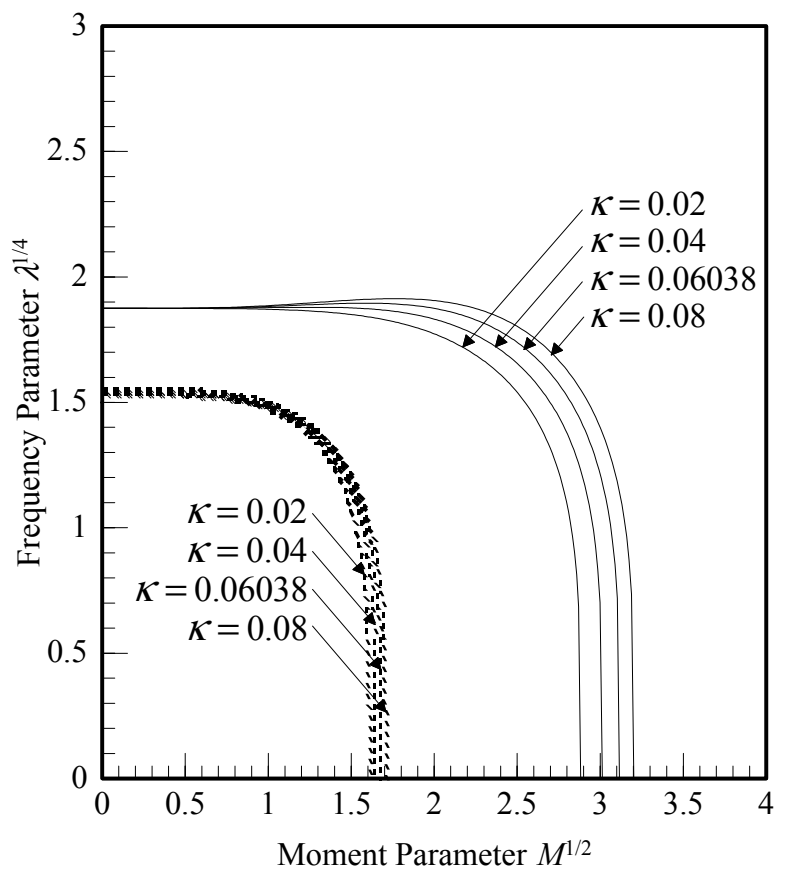

Fig. 4. Effects of warping rigidity on moment buckling (...warping major branch, - bending major branch)

In Fig. 3, it is observed that the axial tension does not affect the warping natural frequency while the bending natural frequency is increased. On the other hand, it increases the static bending buckling moment.

Fig. 4 shows the effects of the normalized warping rigidity ratio $\kappa$ on the $M-\lambda$ interaction due to the constant bending moment alone. $\kappa$ does not affect the bending natural frequency when $M=0$, but it increases the static bending buckling moment. $\kappa$ increases both the torsion-warping natural frequency when $M=0$ and the static torsion-warping buckling load.

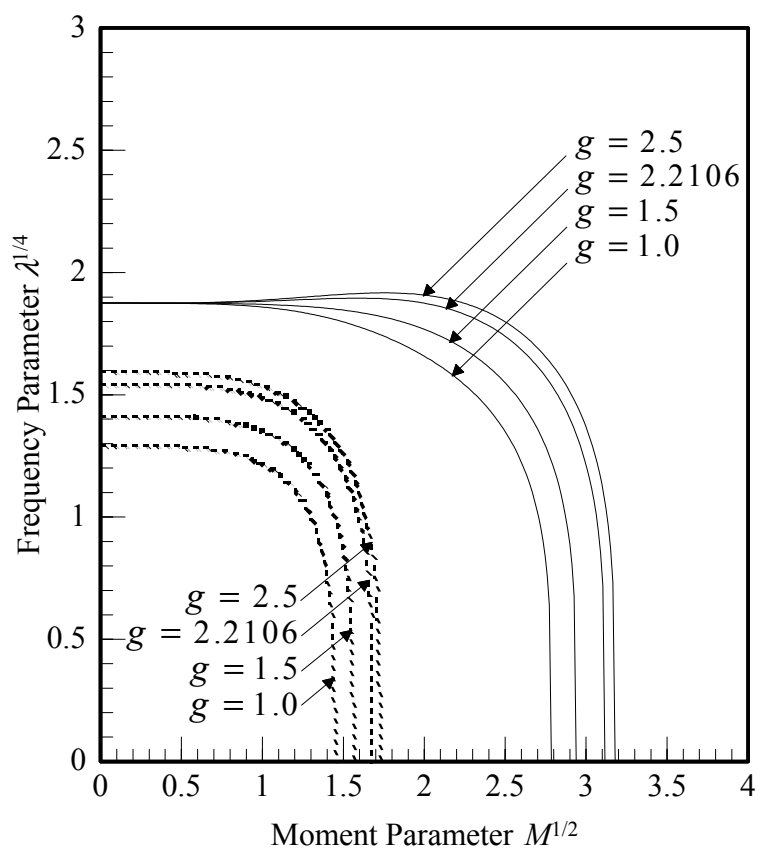

Fig. 5. Effects of warping rigidity on moment buckling (...warping major branch, - bending major branch)

Similarly, Fig. 5 presents the effects of the torsional rigidity ratio $g$ on the $M-\lambda$ interaction due to the constant bending moment alone. $g$ does not affect the bending natural frequency when $M=0$, but it increases the static bending buckling moment. On the other hand, $g$ increases both the torsion-warping natural frequency when $M=0$ and the static torsion-warping buckling load.

\section{CONCLUSION}

The spline finite element method is employed to obtain the dynamic stiffness matrix for the investigation of interactive axial-moment dynamic buckling of linear beam system. The initial axial force and moment can be uniformly or non-uniformly distributed. The moment-frequency interaction diagrams in uniform axial force and moment are plotted. The effects of warping rigidity, torsional rigidity, axial tension and compression are investigated. The method is proved to be very efficient and many interaction diagrams can be plotted easily.

\section{REFERENCES}

[1] I. J. Schoenberg, "Contributions to the problem of approximation of equidistant data by analytic functions. Part A: On the problem of smoothing of graduation. A first class of analytical approximation formulae," Quarterly Applied Mathematics, vol. 4, no. 1, pp. 45-99, 1946.

[2] I. J. Schoenberg, "Contributions to the problem of approximation of equidistant data by analytic functions. Part B: On the problem of osculatory interpolation. A second class of analytical approximation formulae," Quarterly Applied Mathematics, vol. 2, no. 2, pp. 112-141, 1946.

[3] J. X. Gu and S. L. Chan, "A refined finite element formulation for flexural and torsional buckling of beam-columns with finite rotations," Engineering Structures, vol. 27, no. 5, pp. 749-759, 2005.

[4] N. I. Kim, C. C. Fu, and M. Y. Kim, "Stiffness matrices for flexural-torsional/lateral buckling and vibration analysis of thin-walled beam," Journal of Sound and Vibration, vol. 299, no. 4-5, pp. 739-756, 2007. 
[5] A. Y. T. Leung, "Dynamic axial-moment buckling of linear beam systems by power series stiffness," Journal of Engineering Mechanics, vol. 135, no. 8, pp. 852-861, 2009.

[6] A. Y. T. Leung, "Exact dynamic stiffness for axial-torsional buckling of structural frames," Thin-Walled Structures, vol. 46, no. 1, pp. 1-10, 2008.

[7] H. Yang and A. Y. T. Leung, "Coupling dynamic buckling analysis of framed structures using a spline finite element," in Proc. Ninth International Conference on Computational Structures Technology, B.H.V. Topping and M. Papadrakakis, Eds. paper 165, Scotland: Civil-Comp Press, 2008.

[8] S. P. Timoshenko and J. M. Gere, Theory of Elastic Stability, New York: McGraw-Hill Book, 1961.

[9] A. Y. T. Leung, Dynamic Stiffness and Substructures, Berlin: Springer-Verlag, 1993.

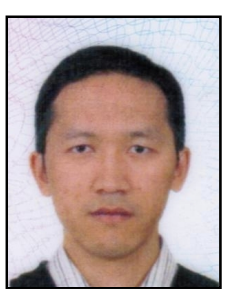

Hao Yang was born in Hubei, China, in 1978. BSc (Eng) degree in Building Service at Faculty of Urban and Environmental Engineering of Shenyang Jianzhu University. Mphil degree in Structural Engineering at Faculty of Civil Engineering of Shenyang Jianzhu University, 2003. PhD in Computational Mechanics at Department of Civil and Architectural Engineering of City University of Hong Kong, 2010. Postdoctoral Fellow at Department of Civil Engineering of The University of Hong Kong. His research interests include structural dynamics, vibration control, and fracture mechanics.

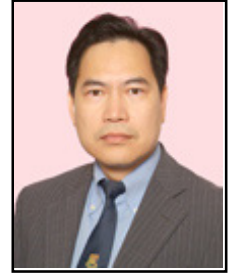

Sai-Huen Lo was born in Hong Kong, China. BSc (Eng), The University of Hong Kong, China. MPhil, The University of Hong kong, China. Doc-Ing, Ecole Nationale des ponts et Chaussees, France. He is professor at Department of Civil Engineering of The University of Hong Kong, SenMIEEE member. His research interests include large deformation problems, structural analysis, finite element mesh generation, adaptive analysis, computer graphics, computer-aided design/analysis, and structural dynamics.

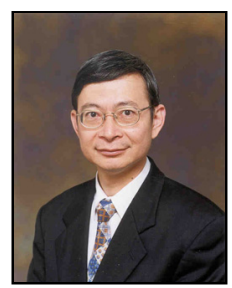

Yee Tak Leung was born in Hong Kong, China. M.Sc. in Mechanical Engineering, Aston University, UK, 1972. Ph.D. in Mechanical Engineering, Aston University, UK, 1976. He is chair professor at Department of Civil and Architectural Engineering of City University of Hong Kong. His research interests include wind and earthquake engineering, fatigue, fracture and corrosion, noise and vibration reduction, monitoring and control, environmental acoustics, structure vibration, dynamic stress, jet fan and machineries, town planning. He published over 600 publications in total, including 7 books and over 300 journal papers 